\title{
Imaging of Acute Aortic Syndromes
}

\author{
Thomas M. Grist and Geoffrey D. Rubin
}

\section{Learning Objectives}

- Understand the pathological classification of acute aortic disease causing acute aortic syndromes.

- Describe eight key imaging findings in the evaluation of acute aortic syndrome.

- Prescribe and implement the correct protocols for accurate diagnosis using $\mathrm{CT}$ and MR imaging of the acute aorta.

The accurate detection and evaluation of acute aortic syndrome is one of the radiologist's most important and immediately impactful opportunities to avoid unnecessary death and disability. Acute aortic syndrome is often a clinical emergency and a situation that demands accurate radiologic diagnosis and intervention to provide lifesaving care. The diagnosis of acute aortic syndrome has evolved significantly over the last two decades, evolving from an arteriographic diagnosis to a diagnosis based upon multi-detector CT angiography [1] and, to a limited extent, MRI. With the advent of these new techniques for diagnosis, investigators have revisited the questions and pathologies surrounding acute aortic syndromes.

\section{T. M. Grist ( $\varangle)$}

Department of Radiology, University of Wisconsin School of Medicine and Public Health, Madison, WI, USA

e-mail: tgrist@uwhealth.org

G. D. Rubin

Department of Radiology, Duke University School of Medicine, Durham, NC, USA

e-mail: grubin@duke.edu

\subsection{Anatomic and Pathological Considerations}

Acute aortic syndromes are principally diseases of the aortic wall. As a result, it is useful to review the fundamental features of the aortic wall in order to categorize the acute aortic syndromes.

The aorta is composed of three layers. From inner to outer, they are the intima, media, and adventitia. The intima is made up of a single layer of flattened epithelial cells with a supporting layer of elastin-rich collagen, fibroblasts, and myointimal cells. The latter myointimal cells tend to accumulate lipid with aging resulting in intimal thickening which is the earliest sign of atherosclerosis. The majority of the aortic wall thickness is composed of the media which itself is broad and elastic with concentric fenestrated sheets of elastin, collagen, and sparsely distributed smooth muscle fibers. The predominance of elastin arrayed as elastic lamina reflects the fact that the aorta and the pulmonary arteries are considered to be the only elastic arteries of the body. Because the aorta and the pulmonary arteries receive the entirety of the cardiac output, they undergo substantial deformation in order to accommodate large volume changes with each cardiac contraction. The remaining arteries of the body are considered muscular arteries with minimal elastin and a predominance of smooth muscle allowing for the body to regulate regional blood flow. The boundary between the intima and media is not readily defined. The division between the intima and media is defined histologically at the internal elastic lamina, which represents the innermost of the many elastic lamellae within the aortic media. The adventitia lacks elastic lamellae and is predominantly composed of loose connective tissue and blood vessels or vasa vasorum.

Anatomically, the aorta can be divided longitudinally into five zones-the aortic root, ascending thoracic aorta, aortic arch, descending thoracic aorta, and abdominal aorta. Acute aortic syndromes can involve any of these five anatomic zones; however they only rarely originate within the abdomi- 
nal aorta. An important principle in the management of acute aortic syndromes is the classification of lesions into Stanford type A or type B. Type A lesions, defined as those involving the ascending aorta or aortic root, are considered lesions that demand urgent surgical intervention with replacement of the diseased ascending aortic segment. The rationale for this urgent intervention is a high risk of aortic rupture, which can lead to pericardial tamponade or frank exsanguination. Type $\mathrm{B}$ lesions do not involve the ascending aorta and as a result can occur within the aortic arch or the descending thoracic aorta. If there is evidence for active aortic rupture, then these patients too should be referred for urgent surgical intervention with endografting becoming an increasingly important option, for treating descending thoracic aortic leaks and pseudoaneurysms [2, 3]. In the absence of active bleeding, they are typically managed with blood pressure reduction and regular monitoring to assess the evolution of aortic dimension and disease extension.

\subsection{Definitions and Classifications}

Collectively, acute aortic syndromes represent lifethreatening conditions that are associated with a high risk of aortic rupture and sudden death. The typical presentation is the sudden onset of chest pain, which may be accompanied by signs or symptoms of hypoperfusion or ischemia to distal organs, extremities, or the brain.

Traditionally, acute aortic syndromes are categorized as aortic dissection, intramural hematoma, and penetrating atherosclerotic ulcer.

Aortic dissection (AD) is characterized by a separation of the aortic media creating an intimal medial complex, which separates from the remaining aortic wall. Blood flowing between the intimal medial complex or flap and the remaining wall is considered to be within a false lumen, whereas blood flow bounded by the intima is considered to be within a true lumen. Multiple communication points can be observed between the true and false lumen. The proximal most communication is considered to be the "entry tear" with the remaining points of communication are considered to be "exit tears" implying flow directionality from true to false lumen and from false to true lumen, respectively. The actual incidence of $\mathrm{AD}$ is difficult to define, since $\mathrm{AD}$ involving the ascending aorta is often a fatal disease and patients frequently die prior to hospitalization. Likewise, AD is sometimes misdiagnosed on initial presentation, and these patients are also at risk for death outside of the hospital. Nevertheless, various population-based studies suggest that the incidence of AD ranges from 2 to 4 case per 100,000 patients [4].

The temporal definition of acute $\mathrm{AD}$ is a dissection that is identified less than 2 weeks after the onset of symptoms, while subacute ranges from 2 to 6 weeks following initial painful episode, and a chronic AD more than 6 weeks after the onset of pain.

Intramural hematoma (IMH) is an entity that was first described approximately 30 years ago as a stagnant collection of blood within the aortic wall. The common association of IMH with pathologically detected intimal defects led to the hypothesis that most are sequelae of penetrating atherosclerotic ulcer. An alternative cause of IMH, typically invoked in the absence of an intimal defect, is rupture of the vasa vasorum. This hypothetical cause of IMH has never been definitively proven. IMH is treated similar to dissections in terms of initial diagnosis as well as clinical management. Most IMH occur in the descending thoracic aorta and can be associated with severe pain. The imaging features vary depending on the amount of blood accumulated in the wall of the aorta, but typically the normal wall measures less than $7 \mathrm{~mm}$ in thickness. The natural history of IMH can be quite variable. Roughly one third of them will progress to aortic dissection, whereas one third will be stable and one third will resolve [5]. The mortality for patients with IMH involving the ascending aorta is similar to that of classic dissection, and therefore these patients are treated as though they have a classic AD with emergent surgery [6]. On the other hand, IMH involving the descending thoracic aorta can be followed, given appropriate therapy for hypertension.

Penetrating atherosclerotic ulcer (PAU) is a condition that originates with atherosclerotic plaque involvement of the aorta, primarily the descending thoracic aorta (Fig. 18.1). As the plaque evolves, the ulceration "penetrates" through the internal elastic lamina into the media of the aortic wall. Overtime the PAU may extend through all three layers of the aortic wall to form a false or pseudoaneurysm. A finding of PAU does not necessarily imply the existence of an acute aortic syndrome. Signs of IMH or extravasation indicate acuity.

There are two limitations to this traditional classification. One concerns the omission of a rupturing true aortic aneurysm, as the nature of the presentation and the severity of the event are similar to that of the other acute aortic syndromes. The other limitation is that IMH, defined as a stagnant intramural collection of blood, can be observed in the setting of $\mathrm{AD}, \mathrm{PAU}$, and rupturing aortic aneurysm. As such, it is a feature or characteristic associated with any of the acute aortic syndromes reflecting degradation of the aortic wall as a harbinger of impending aortic rupture.

In consideration of these two points, a new classification scheme has been proposed based upon the primary location of the lesion within the aortic wall. In this new classification scheme, there are three pathological entities: AD, PAU, and rupturing aortic aneurism [7]. These three entities are differentiated by the fact that AD principally involves the aortic media, PAU originates within the aortic intima, and aortic aneurysm is a disease of all three layers. The presence of IMH is an observation or epiphenomenon to be applied to 

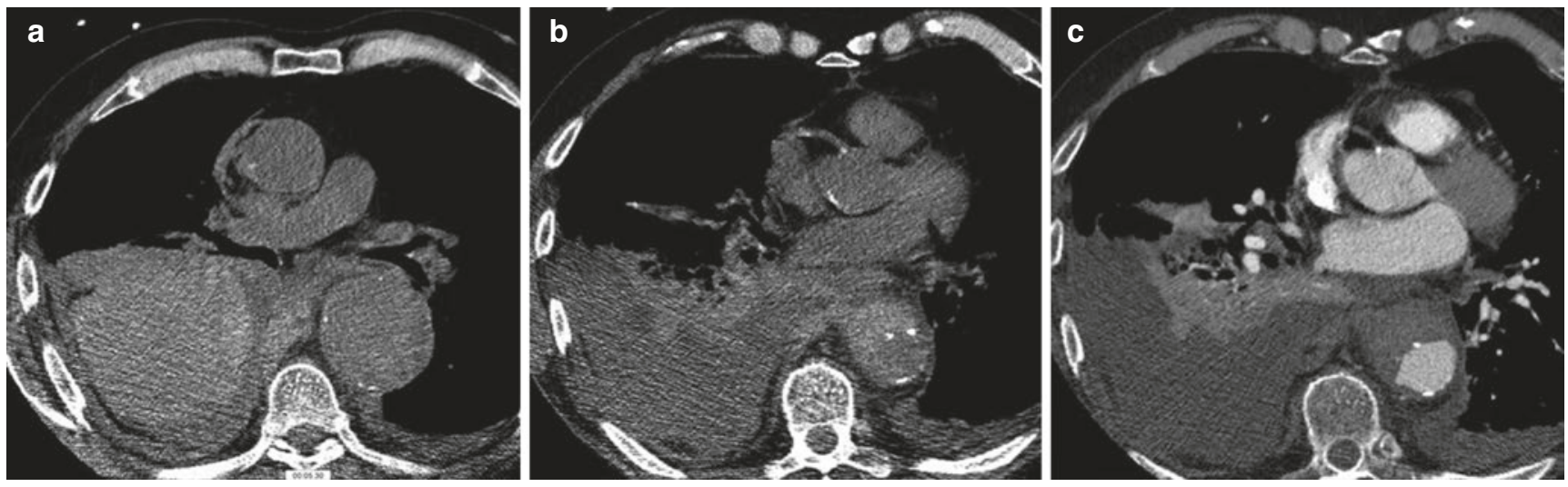

Fig. 18.1 Rupturing thoracic aortic aneurysm: (a) Unenhanced CT demonstrates high-attenuation hematoma in the right pleural space and the middle mediastinum. There is an aneurysm of the descending aorta. (b) Unenhanced CT section $5 \mathrm{~cm}$ inferior to (a) reveals an IMH at the inferior margin of the aneurysm with extravasation of blood into the

any of these three fundamental pathologies. In the setting of an isolated IMH without PAU, "non-communicating dissection" has been proposed as a descriptor, although most people will associate the term "IMH" with this lesion. Recently, a subtype of aortic dissection has been described as "limited intimal tears" [8], which has similar natural history as AD and IMH, but can be particularly subtle to detect given their limited extent along the length of the thoracic aorta.

\subsection{Essential Elements of Aortic Imaging Reports}

In 2010, a group of medical organizations representing the disciplines of cardiology, radiology, thoracic surgery, and anesthesia published guidelines for the diagnosis and management of patients with thoracic aortic diseases. In this report, the authors identified eight essential elements that should be addressed in aortic imaging reports [4]. While these guidelines are not comprehensive, nor do they imply the necessity of reporting every element in every case, they are a useful construct from which to build any formalized description of the imaging findings of an acute aortic syndrome. They are:

1. The location at which the aorta is abnormal

2. The maximum diameter of any dilation, measured from the external wall of the aorta, perpendicular to the axis of flow and the length of the aorta that is abnormal

3. For patients with genetic syndromes at risk for aortic root disease, measurements of aortic valve, sinuses of Valsalva, sinotubular junction, and ascending aorta

4. The presence of internal filling defects consistent with thrombus or atheroma middle mediastinum. Note displacement of intimal calcium along the inner wall of the IMH. (c) Following administration of IV contrast material, the IMH is harder to visualize owing to the wider window used to display the $\mathrm{CT}$ angiogram

5. The presence of IMH, PAU, or calcification

6. Extension of aortic abnormality inter-branch vessels, including dissection and aneurism, and secondary evidence of end-organ injury (e.g., renal or bowel hypoperfusion)

7. Evidence of aortic rupture, including periaortic and mediastinal hematoma, pericardial, and pleural fluid, and contrast extravasation from the aortic lumen (Fig. 18.2)

8. When a prior examination is available, direct image-toimage comparison to determine if there has been any increase in diameter

Recently, predication models have been developed to identify predictors of late adverse events following Type B AD. In particular, false luminal circumferential coverage and maximum aortic diameter at the time of index presentation and a diameter increase of $5 \mathrm{~mm}$ or more in the first 6 months following presentation have been associated with a significant increase in adverse events $[9,10]$.

\subsection{Imaging Approaches to Acute Aortic Syndromes Using CT}

High-quality and comprehensive aortic and end-organ assessment should be performed using multi-detector row CT with at least 16 detector rows. This scanner configuration allows for imaging from the neck through the pelvis, acquiring $\leq 1.5 \mathrm{~mm}$ thick transverse sections during the arterial phase of enhancement from an intravenous contrast administration. It also allows for the use of electrocardiographic gating of the scan when appropriate, as described below. 

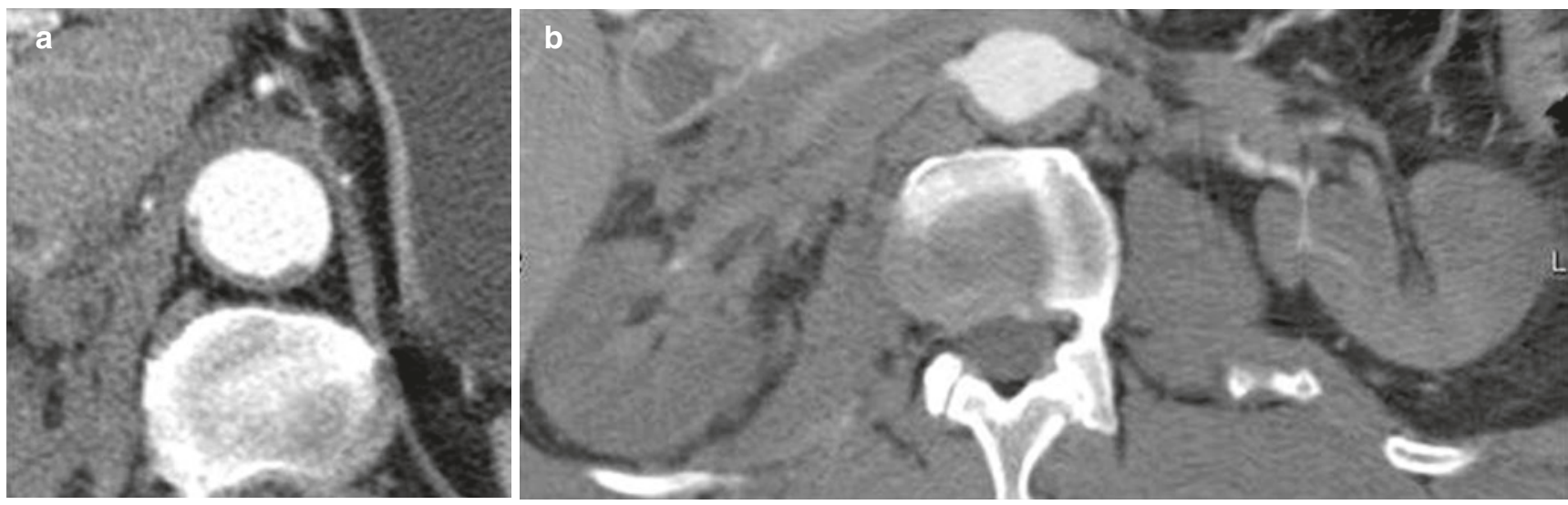

Fig. 18.2 Type B aortic dissection. (a) The true lumen is completely collapsed posteriorly, and only the true lumen fills with contrast material. The entry tear was (not shown) in the proximal descending aorta.

\subsection{Unenhanced CT}

An unenhanced scan can be valuable prior to the administration of intravenous contrast for the detection of what can be subtle intramural and periaortic blood. It can also be useful for mapping the specific regions of the aorta that are abnormal and thus guide the mode of subsequent CT angiographic acquisition. While an associated increase in radiation exposure results from this approach, the potential value of the information almost always outweighs the risk. It has been hypothesized that using dual energy scanning, a virtual unenhanced scan might obviate the need for a separate unenhanced acquisition. However, this approach has not been comprehensively validated in acute aortic syndromes.

\subsection{CT Angiography}

While unenhanced imaging can reveal aortic dilation, intramural and extra-aortic hemorrhage, and in uncommon circumstances directly visualize an intimal flap, the use of intravenously administered contrast medium is required for a complete assessment in suspected acute aortic syndrome (Fig. 18.3). The volume and flow rate of the contrast material should be adjusted based on patient size. A concentrated iodine solution of $\geq 350 \mathrm{mg}$ of iodine $/ \mathrm{ml}$ should be used in order to assure adequate intravenous delivery of iodine with a safe and reliable flow rate of the contrast material into the peripheral vein. Typical volumes and injector flow rates for iodinated contrast range between 60 and $115 \mathrm{~mL}$ at flow rates between 3.5 and $6 \mathrm{~mL} / \mathrm{sec}$.

To assure diagnostic aortic enhancement throughout the CT acquisition, the duration of the contrast injection should exceed the scan duration by $5-10 \mathrm{~s}$, and the initiation of the $\mathrm{CT}$ angiographic acquisition should be based upon the active (b) Because of their supply from the aortic true lumen, the renal arteries do not opacity, and the kidneys are not perfused

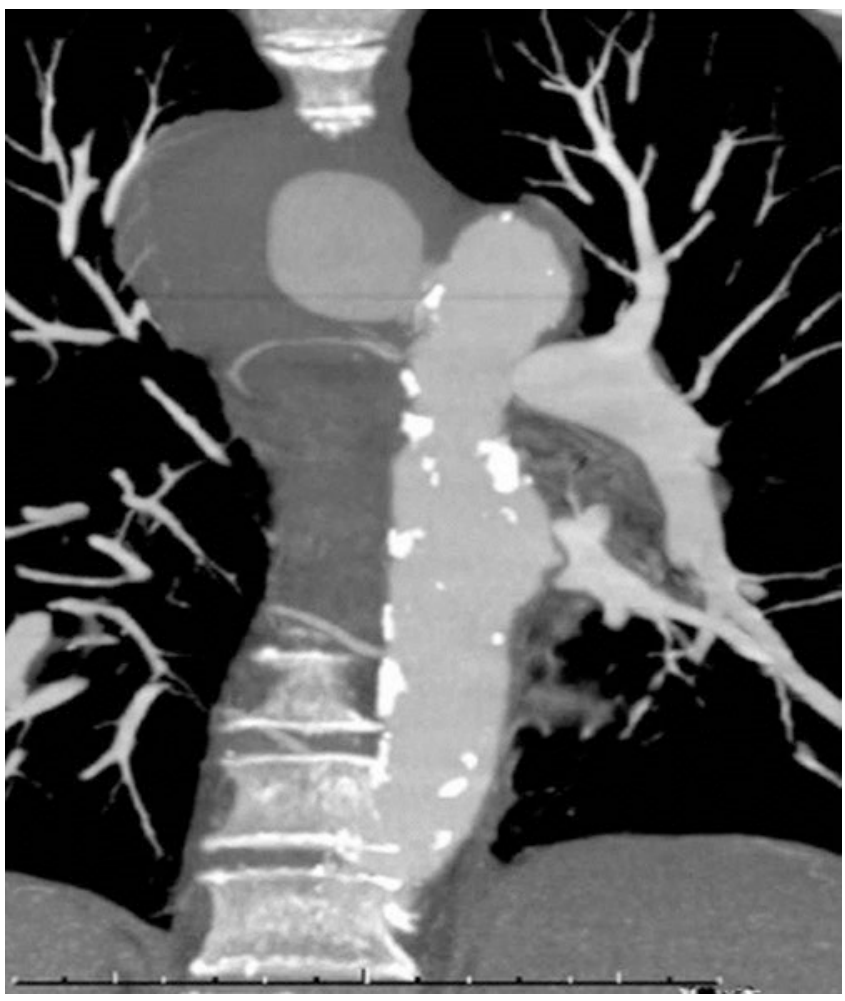

Fig. 18.3 PAU with pseudoaneurysm formation in the proximal descending aorta

monitoring on the arrival of iodine within the descending thoracic aorta.

\subsection{Imaging the Abdominal Aorta and Iliac Arteries}

Because of the likelihood of direct extension of thoracic aortic disease into the abdominal aorta and iliac arteries, unrelated but important abdominal aortoiliac pathology, the value 
of assessing the caliber of a transfemoral delivery route to intra-aortic repair devices, and the possibility for abdominal visceral ischemia, scan ranges that extend through the abdomen and pelvis are highly recommended as a routine approach to imaging acute aortic syndromes. By beginning the scan in the neck and extending inferiorly below the lesser trochanters of the femurs, the scan range will comfortably include several centimeters of the cervical carotid arteries through the bifurcation of the femoral artery. Scan ranges that include less anatomy risk the possibility that important observations will be missed, and additional CT scans with further injections of iodinated contrast material may be required.

\subsection{ECG Gating}

When the ascending aorta is involved with an acute aortic syndrome, electrocardiographic (ECG) gating can be valuable. ECG gating allows for clear delineation of the position of the intimal flap across the cardiac period, distinction of the involvement of the structures of the aortic root including the coronary artery ostia and the aortic annulus, elimination of pulsation-related artifacts that can blur the aortic wall, and subtle regions of extravasation. Unlike the use of ECG gating in the setting of coronary artery disease assessment, the strategy for using ECG gating in acute aortic syndromes does not rely upon the manipulation of heart rate or coronary artery dimension using beta-blockers or nitrates. Regardless of the basal heart rate, the placement of ECG leads and acquisition of a retrospectively gated CT scan (with judicious use of ECG directed X-ray tube current pulsing to minimize radiation exposure) allows for a fourdimensional assessment of the aortic root, aortic valve, coronary arteries, and ascending aorta. It is sufficient to reconstruct ten phases every $10 \%$ of the R-R interval. Gating is only beneficial through the thoracic aorta. For patients with heart rates less than 80 beats per minute, prospective triggering of multiple axial slabs, a single wide area acquisition, or a single high-pitch dual source may suffice to delineate the structures of the aortic root, depending upon the type of CT scanner available. The abdomen and pelvis are acquired after the chest using a non-gated acquisition with minimization of delay between the two scans so that only one contrast injection is required.

ECG gating is not required for all patients; however, it can be critical in patients with aortic root involvement. Review of preliminary unenhanced $\mathrm{CT}$ sections by a radiologist can help to identify patients for whom gating may be of value. Otherwise a second CT scan may be required and could delay treatment.

\subsection{Clinical Indications for MRI in Acute Aortic Syndrome}

While CT remains the mainstay for the initial diagnosis of patients with acute aortic syndrome, MRI may play a limited role in patients who cannot have a contrast-enhanced CTA, as well as in the follow-up of patients for complications following prior aortic dissection. However, due to the immediate availability of non-contrast $\mathrm{CT}$ in most emergency rooms, a non-contrast $\mathrm{CT}$ in patients who have a severe allergy to iodinated contrast may still be the method of choice for evaluating the potential complications of acute aortic syndrome, including IMH, ruptured aorta, pericardial or pleural effusions, and a displaced, calcified intimomedial flap.

Nevertheless, in patients who cannot have iodinated contrast associated with CT, MR may plan a useful role in the diagnosis of patients with acute aortic dissection. Investigators have demonstrated the value of rapid MRI using steady-state free precession (SSFP) for detecting the intimal media flap associated with dissection [11, 12]. Using SSFP, it is possible to perform the diagnostic exam with minimal MRI table time in the acutely ill patient. In addition, contrast-enhanced MRA may be performed quickly and will provide information regarding branch vessel involvement, the presence of intraluminal abnormalities, PAU, and for demonstrating the intimomedial flap location and the entry and exit tears in dissection. We have successfully performed a rapid MR evaluation of the thoracic aorta and great vessels in the acutely ill patient presenting with chest pain in less than 15-minute room time using a rapid MR evaluation protocol in patients presenting to the emergency room [13]. Nevertheless, it should be emphasized the CTA remains the mainstay for evaluating patients with suspected acute aortic syndrome also in part related to the ease of evaluation of the entire thoracic and abdominal aorta.

\subsection{MRI Technique and Imaging Findings}

Evaluation of thoracic pathology with MRI begins with rapid SSFP imaging of the aorta and its major branches (Table 18.1). This technique, when high-performance gradients are used to acquire images using a short repetition time (TR) and short echo time (TE), is especially helpful for imaging aortic vascular pathology in the absence of gadolinium contrast media. These images are typically acquired initially in axial and oblique sagittal projections and gated to the diastolic phase of the cardiac waveform. In addition, for more complete characterization of intimal flap motion in the setting of aortic dissection, cine SSFP MRI can be used to further delineate the entry and exit zones of the intimal media 
Table 18.1 MRI techniques for diagnosis and monitoring AAS

\begin{tabular}{|c|c|c|c|c|c|c|}
\hline Sequence & Plane & TR/TE (MS) & Matrix & Accel factor & Gating & $+\mathrm{Gd}$ \\
\hline \multicolumn{7}{|l|}{ MRI in the acute setting } \\
\hline SSFP & 2D axial/SAG & $3 / 1.5$ & $256 \times 192$ & $1-2$ & Yes & No \\
\hline High-res CE MRA & 3D SAG & $3 / 1$ & $320 \times 256 \times 128$ & 4 & No & $0.1 \mathrm{mmol} / \mathrm{kg}$ \\
\hline \multicolumn{7}{|c|}{ Spin echo for characterization of IMH } \\
\hline $\mathrm{T}_{1}$ fast spin echo & 2D axial & $\sim 600 / 10$ & $256 \times 192$ & $\mathrm{n} / \mathrm{a}$ & No & No \\
\hline $\mathrm{T}_{2}$ fast spin echo & 2D axial & $\sim 2500 / 60$ & $256 \times 192$ & $\mathrm{n} / \mathrm{a}$ & No & No \\
\hline \multicolumn{7}{|c|}{ For characterization of flow abnormalities in complicated subacute or chronic dissection } \\
\hline 4D flow phase contrast & 3D SAG & $10 / 4$ & $256 \times 128 \times 64$ & $6-10$ & Yes & Yes \\
\hline
\end{tabular}

flap, as well as potential dynamic compression of branch vessels. Likewise, cine MRI can be used to further characterize the relationship of a type A dissection flap to the aortic valve and any resultant aortic valve insufficiency.

Gadolinium contrast-enhanced MRA is used to characterize the luminal pathology associated with acute aortic syndrome [14]. Contrast-enhanced MRA is usually acquired during the arterial phase of contrast as well as during the delayed "steady-state" phase of contrast enhancement. Some authors describe the use of time-resolved 3D MRA during a small bolus of GBCA first, thus allowing the radiologist to delineate filling pathways of the true and false lumen and branch vessels dynamically [15]. However, time-resolved images are typically followed by high-resolution 3D images for more precise characterization of the anatomy.

Extracellular Gd contrast agents are the primary diagnostic enhancement agents, and higher relaxivity contrast agents are preferred due to their greater signal at a lower dose as well as their protein binding, which facilitates delayed imaging in the steady state. For delayed imaging, we typically use a fat-suppressed post-contrast $\mathrm{T} 1$-weighted gradient echo image with spoiling of the transverse magnetization. The delayed images are particularly helpful for evaluation of extra luminal pathology affecting the aorta, including aortic leaks, arteritis, and infection, and characterizing the size and extent of hematoma outside of the wall.

The arterial phase contrast-enhanced MRA findings in classic aortic dissection are similar to those identified on CT, including displaced intimal flap, thrombosed lumen, and demonstration of entry and exit tears [12]. One key finding on CT that is not reliable on MR is the displaced intimal calcifications associated with a displaced intimal flap. Contrast-enhanced MRA demonstrates filling patterns in aortic dissection and is helpful in delineating branch vessel involvement and assessing end-organ perfusion.

The exquisite soft tissue contrast in delayed contrastenhanced MR images may be helpful for delineating penetrating aortic ulceration. The immediate arterial phase images on contrast-enhanced MR may show the size and extent of ulceration; however the delayed images add enhanced visu- alization of the aortic adventitia and surrounding soft tissues, thus allowing more definitive characterization of the aortic enlargement and associated structures in penetrating aortic ulceration (Fig. 18.4).

In the setting of suspected intramural hematoma, SSFP MRI or contrast-enhanced MRA alone is inadequate for detecting hemorrhage in the wall of the aorta. In this clinical scenario, T1- and T2-weighted spin-echo technique is typically necessary for diagnosis. MRI using fast spin-echo technique is useful for detecting IMH and characterizing its age or confirming and dating suspected IMH identified on an unenhanced CT scan. IMH exhibits the expected signal characteristics associated with the transition of hemorrhage from deoxyhemoglobin (low T1 and T2) in the hyperacute stage to intracellular methemoglobin (high $\mathrm{T} 1$, intermediate $\mathrm{T} 2$ ) in the subacute stage and extracellular methemoglobin (high T1 and T2) in the late stage of IMH (Fig. 18.5).

Finally, phase contrast MR may be helpful for delineating entry and exit zone sites in classic aortic dissection, as well as for documenting flow patterns in the true and false lumen. In addition, in the setting of suspected branch vessel ischemia, phase contrast MR may demonstrate and quantify the abnormal aortic and branch vessel flow associated with dissection. Future work may allow the use of computational fluid dynamics to predict the likelihood of vascular rupture in patients with aortic aneurysm or dissection; however additional validation of the predictive power of these techniques is necessary.

\subsection{Summary}

High-resolution CT and MR imaging have fundamentally changed the diagnosis and treatment of acute aortic syndrome in the modern era. Multi-detector CTA has streamlined the early diagnosis and management of these patients and has allowed us to rethink the classification of the syndrome as a manifestation of specific pathologies involving the aortic wall. Recent improvements in understanding of this entity contribute to the improved survival with broader 
Fig. 18.4 Penetrating aortic ulcer. (a) Early arterial-phase and (b) delayed "steady-state" phase MRA demonstrating penetrating aortic ulceration involving the superior surface of the aortic arch. Note the excellent delineation of the aortic adventitia on the delayed images due to the contrast enhancement that occurs in the steady-state imaging phase
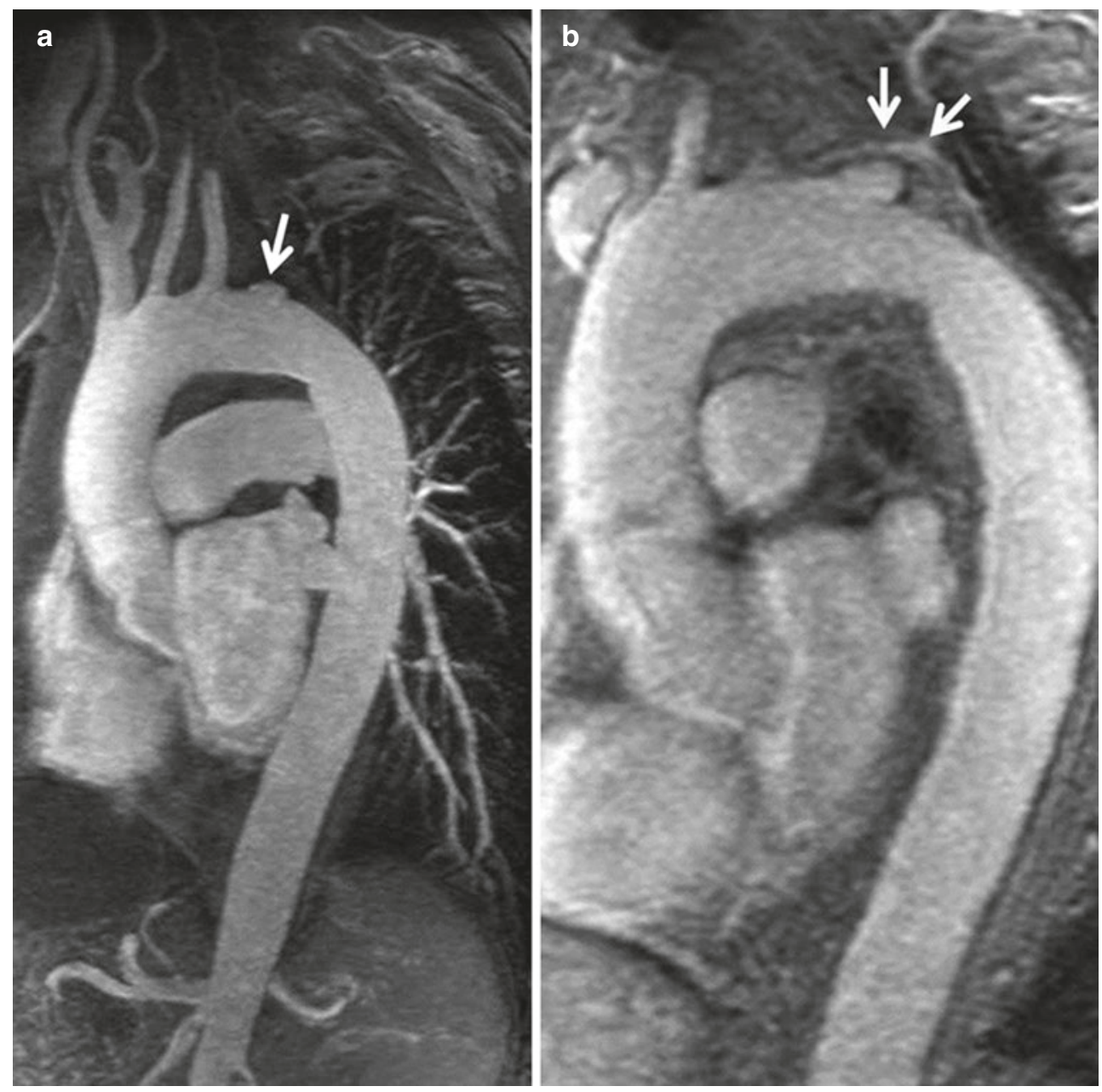
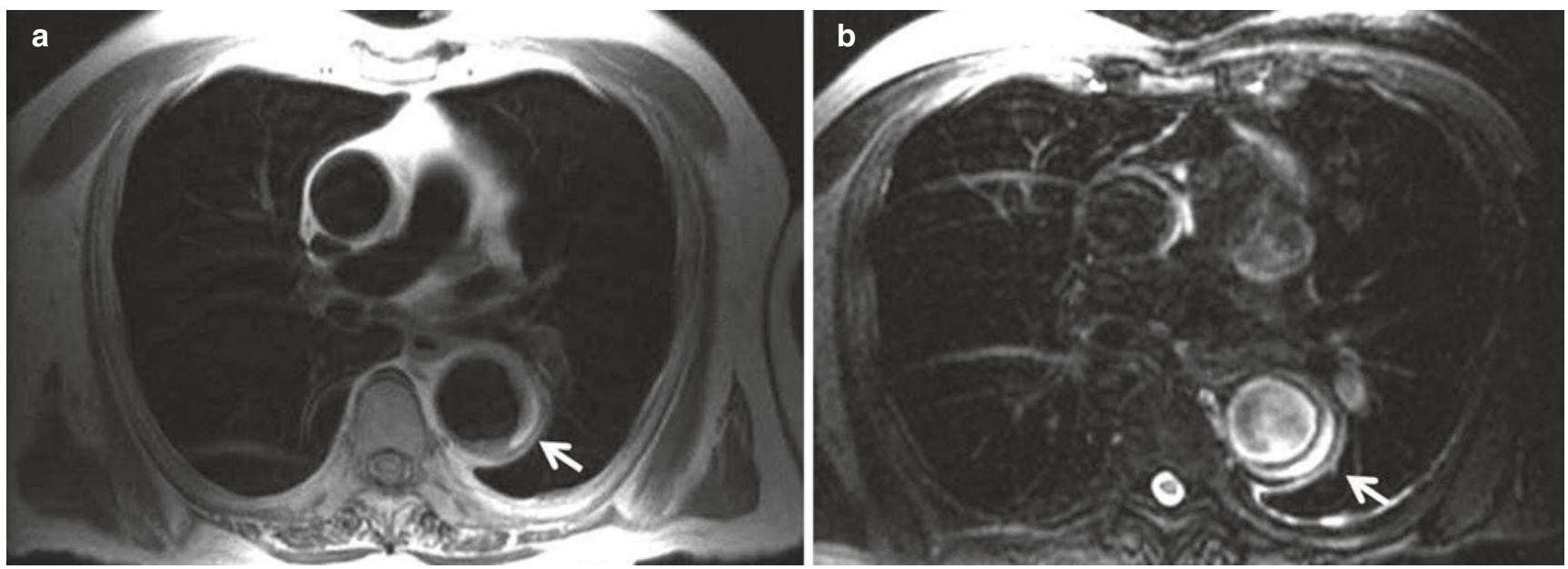

Fig. 18.5 (a) T1-weighted MRI demonstrating IMH with mixed signal intensity components including low signal intensity corresponding to deoxyhemoglobin and high signal intensity extracellular methemoglo- bin suggesting an IMH of subacute or chronic duration. (b) T2-weighted image demonstrating high signal intensity associated with extracellular methemoglobin due to chronic IMH 
treatment options for patients who are correctly diagnosed, including significant advances in endovascular therapy for complicated type B dissections over the last decade. The role of MRI in patients with aortic disease is more limited to the diagnosis and management of complications, follow-up studies, and in the initial diagnosis in patients who cannot undergo CTA. The accurate detection and evaluation of acute aortic syndrome is one of the radiologist's most immediately impactful opportunities to reduce morbidity and mortality associated with this spectrum of diseases.

\section{Key Points}

- A new classification scheme has been proposed based upon the primary location of the lesion within the aortic wall: aortic dissection principally involves the aortic media, penetrating aortic ulcer originates within the aortic intima, and aortic aneurysm is a disease of all three layers.

- The presence of intramural hematoma is an observation or epiphenomenon to be applied to any of these three fundamental pathologies.

- An unenhanced CT scan is important to perform prior to the administration of intravenous contrast for the detection of what can be subtle intramural and periaortic blood and for characterizing the postoperative aorta.

- MRI is useful for specific problem-solving, postdissection surveillance, and hemodynamic assessment in patients with mal-perfusion syndromes.

\section{Take-Home Messages}

- Radiologists can save lives by accurate and immediate diagnosis of acute aortic syndromes.

- Unenhanced and contrast-enhanced CTA is the quickest, most available, and accurate method to determine the pathological cause of acute aortic syndrome.

- An understanding of the pathological origin of aortic disease is important to accurate description of the key imaging findings identified in the setting of acute aortic syndrome.

\section{References}

1. Rubin GD, Leipsic J, Joseph Schoepf U, Fleischmann D, Napel S. CT angiography after 20 years: a transformation in cardiovascular disease characterization continues to advance. Radiology. 2014;271(3):633-52.

2. Arafat A, Roselli EE, Idrees JJ, et al. Stent grafting acute aortic dissection: comparison of DeBakey extent IIIA versus IIIB. Ann Thorac Surg. 2016;102(5):1473-81.

3. Geisbusch P, Kotelis D, Weber TF, Hyhlik-Durr A, Kauczor HU, Bockler D. Early and midterm results after endovascular stent graft repair of penetrating aortic ulcers. J Vasc Surg. 2008;48(6):1361-8.

4. Hiratzka LF, Bakris GL, Beckman JA, et al. 2010 ACCF/AHA/ AATS/ACR/ASA/SCA/SCAI/SIR/STS/SVM guidelines for the diagnosis and management of patients with thoracic aortic disease: a report of the American College of Cardiology Foundation/ American Heart Association task force on practice guidelines, American Association for Thoracic Surgery, American College of Radiology, American Stroke Association, Society of Cardiovascular Anesthesiologists, Society for Cardiovascular Angiography and Interventions, Society of Interventional Radiology, Society of Thoracic Surgeons, and Society for Vascular Medicine. Circulation. 2010;121(13):e266-369.

5. Ganaha F, Miller DC, Sugimoto K, et al. Prognosis of aortic intramural hematoma with and without penetrating atherosclerotic ulcer: a clinical and radiological analysis. Circulation. 2002;106(3):342-8.

6. Evangelista A, Mukherjee D, Mehta RH, et al. Acute intramural hematoma of the aorta: a mystery in evolution. Circulation. 2005;111(8):1063-70.

7. Fleischmann D, Mitchell RS, Miller DC. Acute aortic syndromes: new insights from electrocardiographically gated computed tomography. Semin Thorac Cardiovasc Surg. 2008;20(4):340-7.

8. Chin AS, Willemink MJ, Kino A, et al. Acute limited intimal tears of the thoracic aorta. J Am Coll Cardiol. 2018;71(24):2773-85.

9. Sailer AM, van Kuijk SMJ, Nelemans PJ, et al. CT imaging features in acute uncomplicated stanford type-B aortic dissection predict late adverse events. Circ Cardiovasc Imaging. 2017;10(4):e005709.

10. Sailer AM, Nelemans PJ, Hastie TJ, et al. Prognostic significance of early aortic remodeling in acute uncomplicated type B aortic dissection and intramural hematoma. J Thorac Cardiovasc Surg. 2017;154(4):1192-200.

11. Pereles FS, McCarthy RM, Baskaran V, et al. Thoracic aortic dissection and aneurysm: evaluation with nonenhanced true FISP MR angiography in less than 4 minutes. Radiology. 2002;223(1):270-4.

12. Gebker R, Gomaa O, Schnackenburg B, Rebakowski J, Fleck E, Nagel E. Comparison of different MRI techniques for the assessment of thoracic aortic pathology: 3D contrast enhanced MR angiography, turbo spin echo and balanced steady state free precession. Int J Cardiovasc Imaging. 2007;23(6):747-56.

13. Schiebler ML, Nagle SK, Francois CJ, et al. Effectiveness of MR angiography for the primary diagnosis of acute pulmonary embolism: clinical outcomes at 3 months and 1 year. J Magn Res Imaging. 2013;38(4):914-25.

14. Prince MR, Narasimham DL, Jacoby WT, et al. Three-dimensional gadolinium-enhanced MR angiography of the thoracic aorta. AJR Am J Roentgenol. 1996;166(6):1387-97.

15. Finn JP, Baskaran V, Carr JC, et al. Thorax: low-dose contrastenhanced three-dimensional MR angiography with subsecond temporal resolution - initial results. Radiology. 2002;224(3):896-904.

Open Access This chapter is licensed under the terms of the Creative Commons Attribution 4.0 International License (http://creativecommons. org/licenses/by/4.0/), which permits use, sharing, adaptation, distribution and reproduction in any medium or format, as long as you give appropriate credit to the original author(s) and the source, provide a link to the Creative Commons license and indicate if changes were made.

The images or other third party material in this chapter are included in the chapter's Creative Commons license, unless indicated otherwise in a credit line to the material. If material is not included in the chapter's Creative Commons license and your intended use is not permitted by statutory regulation or exceeds the permitted use, you will need to obtain permission directly from the copyright holder. 